range of vascular pathologies including vascular inflammation and atherosclerosis. While TNF- $\alpha$ has shown to regulate Tie receptors, the chronic impact IL-1 $\beta$ has on Ang-1/Tie receptor signalling pathway and vascular function has not been investigated.

Aim To examine the impact IL- $1 \beta$ has on Tie receptor expression and Angiopoietin-1 induced PI3Kinase/AKT activity in endothelial cells.

Method Primary Human Umbilical Vein Endothelial Cells (HUVEC) were stimulated with $25 \mathrm{ng} / \mathrm{ml}$ of IL- $1 \beta$ in the presence or absence of $100 \mathrm{ng} / \mathrm{ml}$ of human recombinant Ang-1. The treatment times ranged from 0 to $48 \mathrm{~h}$. Cell lysates from the treated cells were then subjected to Western blotting to analyze Tie receptor levels and phospho-AKT (pAKT), a signaling molecule associated with Ang-1 cellular transduction. The levels of target proteins were compared between reactions by quantifying mean intensity of bands.

In addition, cells were treated with Ang-1 in the absence or presence of IL-1 $\beta$ at various time points. The cell viability assay was performed on the treated cells by following the manufactures protocol. The mean percentage of live to dead cells was calculated from three random fields for each treatment.

Data for the Tie receptor and AKT analysis is presented as means and SEM of three independent experiments. Statistical significance represented with $\mathrm{p}<0.05$ using Student's t-test. Data for the cell viability assay is presented as means and SEM of two independent experiments.

Results A significant reduction in the levels of Tie-1 receptor was observed at $3 \mathrm{~h}(58.6 \pm 8.6 \%)$ in HUVECs treated with IL$1 \beta$. The cytokine was able to maintain significant low levels of Tie1 up until $48 \mathrm{~h}$ of treatment, whereas the changes in levels of Tie- 2 were insignificant. Interestingly, IL-1 $\beta$ significantly reduced Ang1-induced pAKT activity from 3h onwards with maximum reduction of $73.4 \pm 12.8 \%$ observed at $48 \mathrm{~h}$. The cell viability assays showed reduction in the percentage live to dead cells between Ang-1 and Ang1 + IL-1 $\beta$ for chronic time points tested.

Conclusion Long term exposure of Interleukin-1 $\beta$ is capable of altering Tie-1 levels and increasing the Tie-2: Tie-1 ratio in endothelial cells. In contracts, IL-1 $\beta$ reduces Ang-1-PI3Kinase/ AKT signalling and endothelium cell viability. This opposing phenomenon observed where IL-1 $\beta$ impairs Ang- 1 protective ability suggests and different mechanism of regulation, independent of the Tie-1 receptor.

Conflict of Interest None

\section{BS21 STABILISATION OF SUPPRESSOR OF CYTOKINE SIGNALLING 3 (SOCS3) TO INHIBIT HUMAN SAPHENOUS VEIN SMOOTH MUSCLE CELL PROLIFERATION AND VASCULAR STENOSIS}

${ }^{1}$ Tim Palmer, ${ }^{2}$ Florah Moshapa, ${ }^{3}$ Jamie Williams, ${ }^{2}$ Kirsten Riches-Suman, ${ }^{2}$ Jacobo Elies. ${ }^{1}$ University of Hull, Hull, UK; ${ }^{2}$ University of Bradford; ${ }^{3}$ University of Glasgow

10.1136/heartjnl-2021-BCS.219

Introduction Suppressor of cytokine signalling 3 (SOCS3) limits multiple signalling pathways involved in vascular inflammation and remodelling responsible for neointimal hyperplasia and vein graft failure. However, SOCS3 function is limited by its short biological half-life, suggesting that SOCS3 stabilisation might prove an effective therapeutic strategy. Through identification of SOCS3 ubiquitination sites, we have engineered a novel SOCS3 transgene resistant to proteasomal degradation and assessed its ability to limit signalling pathways and processes responsible for neointimal hyperplasia.

Methods Flag-tagged SOCS3 transiently expressed in HEK293 cells was immunoprecipitated with anti-Flag antibody and fractionated by SDS-PAGE for trypsin digestion. Tryptic peptides were analysed by liquid chromatography and tandem mass spectrometry, and ubiquitination sites identified from di-Glymodified SOCS3 peptides. Smooth muscle cells (SMCs) and endothelial cells (ECs) from human saphenous vein (HSV) were transduced with recombinant lentiviruses (LVs) with MOIs ranging from 3.6-22.2 tfu/cell. Ubiquitination was assessed by immunoprecipitation of soluble cell lysates and immunoblotting. Half-lives of SOCS3 transgenes were determined by immunoblotting following HSVSMC incubation \pm protein synthesis inhibitor emetine. HSVSMC proliferation was assessed by cell counting. Finally, effects of WT and Lysless SOCS3 gene delivery on cell signalling was determined by assessing phosphorylation of STAT3 (Tyr705) and ERK1/2 (Thr202/Tyr204) by immunoblotting.

Results Analysis of recombinant SOCS3 immunoprecipitated from HEK293 cells revealed that 8 of the 9 Lys residues in human SOCS3 were ubiquitinated. A mutated SOCS3 in which all 9 Lys residues were mutated to Arg was resistant to ubiquitination compared to wild type (WT) SOCS3. LV transduction of WT and Lys-less SOCS3 in HSVSMCs and ECs was highly efficient with $>90 \%$ of cells expressing SOCS3 transgene after 48 hours. Lys-less SOCS3 was significantly more stable, displaying a biological half-life $>4$ hours versus $<4$ hours for WT $(n=6, p<0.001)$. Despite these differences, WT and Lys-less SOCS3 were functionally equivalent in their ability to selectively inhibit STAT3 phosphorylation in response to either sIL-6R $\alpha / \mathrm{IL}-6 \quad(74 \pm 6 \%$ and $80 \pm 7 \%$ inhibition respectively; $\mathrm{n}=4, \mathrm{p}<0.05$ versus controls) or PDGF-BB $(67 \pm 17 \%$ and $72 \pm 18 \%$ inhibition respectively; $n=5)$ but not ERK1/2 phosphorylation. However, while WT and Lys-less SOCS3 each inhibited cell proliferation in response to sIL$6 \mathrm{R} \alpha / \mathrm{IL}-6(83 \pm 29 \%$ and $89 \pm 22 \%$ inhibition respectively; $n=4$, $\mathrm{p}<0.05$ versus controls) only Lys-less SOCS3 significantly inhibited PDGF-BB-stimulated proliferation $(67 \pm 11 \%$ inhibition; $\mathrm{n}=4, \mathrm{p}<0.05$ versus controls).

Conclusions These results provide evidence for possible therapeutic targeting of SOCS3 stabilisation to limit HSVSMC dysfunction responsible for vein graft failure.

Conflict of Interest None

\section{BS22 TRPV4 IS A PHOSPHOLIPASE C COUPLED RECEPTOR WHEN MITOCHONDRIA ARE DEPOLARISED IN INTACT ENDOTHELIAL CELLS}

XUN ZHANG, Calum Wilson, John McCarron. University of Strathclyde, Glasgow, UK

\subsection{6/heartjnl-2021-BCS.220}

Mitochondria are significant regulators of intracellular $\mathrm{Ca} 2+$ signalling in the vascular endothelium. Depolarization of the mitochondrial membrane potential $(\Delta \psi \mathrm{m})$ inhibits IP3-mediated $\mathrm{Ca} 2+$ release and may regulate $\mathrm{Ca} 2+$ influx across the plasma membrane. However, precisely how $\mathrm{Ca} 2+$ influx is regulated by mitochondria in the endothelium is unknown. To examine mitochondrial regulation of $\mathrm{Ca} 2+$ influx, the interaction of TRPV4-mediated $\mathrm{Ca} 2+$ influx with 